\title{
VALORIZAR A EXPERIÊNCIA DE VIDA E DE TRABALHO NA EDUCAÇÃO E FORMAÇÃO - BREVES SUBSÍDIOS AO DEBA- TE
}

\author{
Rosanna Barros \\ E-mail: rmbarros@ualg.pt \\ Universidade do Algarve (Portugal) \\ DOI: 10.15628/rbept.2020.11780
}

Artigo submetido em: dez/2020 e aceito em: dez/2020

\section{RESUMO}

Este artigo, de caráter ensaístico, procura convocar breves diacríticas inerentes à corrente das histórias de vida nas ciências da educação e formação, para melhor equacionar o papel da experiência de vida e de trabalho no âmbito dos processos educacionais que envolvem jovens e adultos, quer se trate dos contextos formais quer se trate dos contextos não-formais da educação e formação. Com este enquadramento, reflete-se acerca de alguns aspetos referentes à contraposição entre o diagnóstico de necessidades e o reconhecimento dos adquiridos experienciais.

Palavras-chave: Educação e Formação de jovens e adultos. Histórias de vida. Diagnóstico de necessidades. Reconhecimento dos adquiridos experienciais.

\section{VALORIZATION OF LIFE AND WORK EXPERIENCE IN TRAI- NING AND EDUCATION: PERSPECTIVES FOR DEBATE}

\begin{abstract}
This article, of an ensaistic nature, search to call some brief diacritics inherent to life histories in the sciences of education and training, to better equate the role of life and work experience in the context of educational processes involving young people and adults, whether it is formal contexts or the non-formal contexts of education and training. With this framework, we reflect about some aspects related to the opposition between the diagnosis of needs and the recognition of prior experiential learning.
\end{abstract}

Keywords: Youth and adult education. Life's history. Needs diagnosis. Acquired experiential. 


\section{CONSTRUÇÃO DE SENTIDO(S) ENTRE VIDA-TRABALHO-EDUCAÇÃO}

Neste artigo abordamos a relação entre Educação e Formação (de jovens e adultos) e a construção de identidade(s) pela experiência de vida e de trabalho. Neste âmbito, optámos por refletir acerca de alguns aspetos referentes à contraposição entre o diagnóstico de necessidades e o reconhecimento dos adquiridos experienciais. Trata-se de uma problemática que coloca no centro do debate a questão da adequação contextualizada dos processos e ofertas de educação e formação de jovens e adultos (de qualquer nível escolar), conferindo importância à experiência de quem aprende, numa lógica já não de rutura mas sim de articulação com os contextos em que se desenrola a vida e o trabalho do sujeito aprendente. O sujeito ganha, pois, uma centralidade que não era possível no âmbito do paradigma escolar tradicional, reequacionando-se, no seio deste debate, o papel e estatuto que os saberes prévios, a uma dada situação de aprendizagem, têm na produção de novos conhecimentos. Ou seja, tomam-se retrospetivamente os percursos já realizados de maneira a identificar as principais capacidades e saberes já adquiridos, procurando-se principalmente evitar o erro de pretender ensinar conteúdos que as pessoas já dominam.

Neste tipo de abordagem teórico-pedagógica parte-se do pressuposto de que as aprendizagens experienciais, resultantes do percurso de vida, são tão ou mais decisivas para a concretização de novas aprendizagens como as aprendizagens formais derivadas do percurso escolar (e traduzidas por um certificado). No entanto, a forma como se trabalha com este material experiencial é substantivamente distinta quando se está na ótica da conceção e prática que se estrutura a partir do levantamento ou diagnóstico das necessidades, ou quando se está na ótica da conceção e prática que se alicerça em torno da valorização ou reconhecimento de adquiridos experienciais. Ou seja, na organização deliberada de práticas de educação e formação (de jovens e adultos) convocam-se, em ambas as situações, os saberes adquiridos experiencialmente, mas sob pressupostos teórico-conceptuais quase antagónicos, na medida em que, num caso se adota uma lógica geral que procura visibilizar sobretudo os défices e as lacunas que os sujeitos possuem, enquanto que no outro caso o que se procura é evidenciar o que já está presente, isto é, o que o sujeito já possui como aquisições.

De modo geral, no campo da educação e formação (de jovens e adultos) o diagnóstico das necessidades de formação nasceu e manteve-se associado, sobretudo, ao mundo económico e empresarial por via de uma formação profissional contínua, em que prevalece tendencialmente os pressupostos teórico-conceptuais da teoria da formação dos recursos humanos, onde predominam conceitos como os de qualidade, eficácia e engenharia da formação. Por seu turno, os procedimentos relacionados com o reconhecimento 
dos adquiridos experienciais surgiram inicialmente associados a dois contextos históricos particulares do pós-guerra em que as pressões de grupos de interesses específicos garantiriam o reconhecimento das experiências vividas fora do percurso escolar como forma de assegurar o acesso ao ensino superior, nomeadamente por parte de grupos de soldados norte-americanos, e por parte de grupos feministas canadianos (BARROS, 2011A; 2011B).

Desta forma, nasceria a construção de dois tipos de oferta formativa (para jovens e adultos) que, principalmente na sua origem, advogam visões distintas em termos da conceção dos sujeitos e dos procedimentos pedagógicos a adotar para tomar em linha de conta a experiência prévia de quem aprende. Predominando num caso a metodologia da análise de necessidades e no outro caso a metodologia do balanço de competências.

Acerca da análise de necessidades, em que se alicerça a abordagem teórico-pedagógica da formação profissional contínua, há a assinalar sobretudo que os seus pressupostos de base tendem a ignorar, quer o facto de existir uma imprevisibilidade intrínseca aos resultados de qualquer ação formativa, quer a impossibilidade de captar, através de inquéritos individuais que expressam os pedidos de formação, a real inserção social dos atores nos contextos em que se desenrolam as suas experiências e, por conseguinte, em que se evidenciam as suas "lacunas" de formação. Trata-se, na maior parte dos casos, de uma prática assente numa perspetiva instrumental e numa racionalidade técnica de que resultam, sobretudo, ações de formação de carácter pontual e de capacitação individual, elaboradas na base de necessidades que são, na verdade, mais o resultado de uma construção social do que de uma real necessidade de formação (BARBIER; LESNE, 1986), isto, na medida em que, o próprio conceito de necessidade de formação é atravessado por diversas ambiguidades que o tornam profundamente problemático, apesar de se manter ainda dominante.

Parece-nos relevante salientar que a controvérsia instalada no âmbito do debate gerado pela problemática que opõe o diagnóstico de necessidades de formação ao reconhecimento de adquiridos experienciais tem como eixo principal a ideia de que no âmbito da prática do reconhecimento dos adquiridos se propiciam como principais recursos de uma prática, que pretende ser também formativa, a personalidade e a experiência dos próprios sujeitos que ao identificar os adquiridos experienciais deverão ser capazes, ajudados por uma metodologia específica, de reelaborar os seus saberes integrando-os e transferindo-os para situações e contextos diversos nos quais se desenrola o processo de autoconstrução da pessoa (CANÁRIO, 2000). Trata-se, como refere Malinen (2000), de procurar operacionalizar uma articulação entre experiência e reflexão, partindo de alguns dos pressupostos do construtivismo piagetiano e do ciclo de aprendizagem conceptualizado por Kolb (1984). 
Ora, o impacto desta viragem conceptual no âmbito das práticas de educação e formação de jovens e adultos tem-se feito sentir, especialmente, desde o último quartel do século vinte (BEARD; WILSON, 2002). Os pressupostos pedagógicos orientadores da ação educativa, particularmente na modalidade da educação não formal, têm traduzido a transição de uma lógica de rutura com a experiência dos sujeitos para uma lógica de continuidade com a sua experiência anterior em que ganha relevo o conceito de aprendizagem experiencial (MIETTINEN, 2000; MOON, 2004; WESSELS, 2006; SILBERMAN, 2007). Há inclusive toda uma revalorização epistemológica da experiência patente no corpus teórico do campo, que tem vindo a contribuir, de igual modo, para a construção de uma maior autonomia teórica da educação e formação de jovens e adultos. Destacando-se, de forma especial, neste contexto, a corrente das histórias de vida, entendidas concomitantemente como instrumentos de investigação, de formação e de intervenção.

\section{A EXPERIÊNCIA PRÉVIA DE QUEM APRENDE - NOTA TEÓRICO-CON- CEPTUAL}

A reflexão teórico-pedagógica acerca das perspetivas biográficas no campo da educação e formação de jovens e adultos, usadas enquanto instrumento de investigação, de intervenção e de formação, ficaria a dever-se, em grande medida, ao trabalho pioneiro desenvolvido pelos teóricos da corrente das histórias de vida em educação da escola de Genebra, que representa um dos polos principais deste movimento nos países francófonos. Muito embora, também nos Estados Unidos, se tivessem desenvolvido, paralelamente, importantes trabalhos que equacionavam as questões da reflexão e da experiência nos processos de aprendizagem dos adultos, que destacavam já a importância do sujeito, mas por uma via distinta, com origem em John Dewey e particularmente nas leituras que dele fez Donald Schön (NÓVOA, 2002).

Um aspeto que os teóricos da escola de Genebra têm acentuado, com particular insistência, é que não chega que se adapte as teorizações vindas do terreno das ciências sociais e humanas, em especial da sociologia, para um domínio com as características do campo educacional, sendo fundamental que se procure descobrir e reinventar o potencial das perspetivas biográficas para as problemáticas de educação e formação de jovens e adultos. Esta corrente de pensamento tem vindo, com efeito, a conceptualizar as histórias de vida em educação como projeto de conhecimento e como projeto de formação, um tipo de formulação teórica que confere à escola de Genebra um estatuto fundador, na medida em que "a dinâmica de articulação entre formação e investigação faz da nossa abordagem biográfica uma versão inovadora 
do método das histórias de vida no campo das ciências humanas" (DOMINICÉ, 1990, p. 71).

A prática das histórias de vida em educação e formação de jovens e adultos tem vindo a ser entendida, desde os contributos da corrente de Genebra, como uma mediação particularmente adequada às complexas características contemporâneas das sociedades ocidentais, expressas sob a forma de múltiplos riscos e incertezas que, no entender de Zygmunt Bauman (2001), configuram para os dias de hoje uma modernidade líquida. Isto sobretudo porque, como sublinha Josso, "a segmentação do 'saber-viver' operada pela sociedade industrial para a comodidade de uma divisão social do trabaIho e dos poderes que Ihe estão ligados (...) e o paradigma cartesiano do conhecimento pela fragmentação [provocam] no 'sendo' que somos um sentimento de divisão e de perda de uma compreensão da nossa existencialidade" (JOSSO, 2002, p. 119). Aliás, este é um dos fatores que, segundo esta corrente, justifica plenamente a criação da prática das histórias de vida em educação e formação, nos termos em que vem sendo desenvolvida pela escola de Genebra, e que se orienta no sentido de fomentar uma "consciência reunificada de nós mesmos, individual e coletivamente" (id., ibid.), apresentandose e assumindo-se enquanto uma prática crítica que opera um "deslocamento para uma posição meta-disciplinar na qual a busca de um 'saber-viver' ou a procura de uma sabedoria tenta uma reintegração operante dos conhecimentos no seio da nossa existencialidade" (id., ibid.).

A este respeito Nóvoa afirma que "a interrogação epistemológica que atravessa hoje em dia alguns círculos da formação de adultos tem origem numa crítica a uma 'visão desenvolvimentista' da educação e na procura de uma conceção da formação que permita ao indivíduo 'pensar-se na ação"” (NÓVOA, s/d, p. 70). Trata-se de ensaiar um entendimento novo da educação e formação de jovens e adultos, que prescinda dos tradicionais conceitos de progresso e de desenvolvimento, repensando as questões e as problemáticas principais da educação e formação a partir do potencial contido nas perspetivas biográficas. Assim, a educação e formação ao longo da vida, terá de considerar na sua prática educativa presente para além da ideia de desenvolvimento futuro também a ideia de retrospeção, ideias interrelacionadas na praxis educacional com base numa metodologia de balanço de vida. Considerando o essencial destes pressupostos Nóvoa sublinha que "o conceito de reflexividade crítica deve assumir um papel de primeiro plano no domínio da formação de adultos (...) o adulto está implicado numa ação presente, o que o obriga a ter em conta um outro vetor dominante da formação de adultos, a consciência contextualizada" (id., ibid., p. 72).

É de resto, no âmbito desta conjuntura, que a problemática da construção das identidades dos sujeitos, da valorização das suas subjetividades, bem como a problemática do papel dos atores na construção das representações sociais, tem vindo a ganhar cada vez mais um maior relevo no campo 
da educação e formação de jovens e adultos, ao nível das práticas educativas e das correspondentes formulações teóricas (BARROS; LIMA; AZEVEDO, 2020). Uma realidade que traduz o que se passa no âmbito das próprias ciências sociais e humanas, domínio em que se destacam, desde os anos oitenta, um conjunto de novas preocupações nesta matéria, por exemplo, em torno do que se conceptualizou como o retorno do ator, ou em torno de uma maior articulação entre as esferas micro e macro estruturais da sociedade (TOURAINE, 1984; GIDDENS, 1991, SOUSA; SANTOS, 2000).

Uma vez que a pesquisa identitária e a consideração contextualizada do sujeito se encontram presentemente no coração da praxis da investigação social, não surpreende que no contexto da reflexão educacional que envolve jovens e adultos se verifique, igualmente, um crescente esforço para analisar qualitativamente os mesmos aspetos singulares implicados na praxis educativa que, assim, reassume e reconfigura a relação entre trabalho e educação. Esta preocupação encontra-se espelhada na literatura que tem surgido mais recentemente no sector, normalmente através de conceptualizações inscritas em matrizes teóricas diversas que giram em torno dos designados processos experienciais (Itin,1999; Baker; Jensen; Kolb, 2002). Processos experienciais que nas dinâmicas interativas de educação e formação e nos processos de intervenção, superam os pressupostos do inquérito clássico, pois, como refere Dionne trata-se de "uma abordagem empírica que valoriza de antemão o ator, na sua relação consigo mesmo e com o ambiente social, obrigando o investigador-interventor a realizar práticas mais consequentes de desenvolvimento, e também mais socializantes quanto aos resultados alcançados" (DIONNE, 1996, p. 181). Ora, o essencial do impacto para a educação e formação de jovens e adultos deste tipo de abordagem está relacionado com o reconhecimento da centralidade da pessoa nos processos de educação e de formação. Este reconhecimento tem-se vindo a afirmar progressivamente no sector, por um lado, com as propostas de construção de um estatuto epistemológico do sujeito, que englobe a consideração consequente da sua subjetividade nos processos de construção de conhecimento, e por outro lado, com a revalorização epistemológica da experiência e a sua consideração consequente nos processos de aprendizagem (BOLIVAR, DOMINGO; FERNÁNDEZ, 2001).

\section{EDUCAÇÃO E FORMAÇÃO COMO PRÁTICA AUTOPÓIETICA - A (RE)CONSTRUÇÃO ATIVA DE IDENTIDADE(S)}

Assim, no geral, a prática das histórias de vida em educação e formação assenta numa proposta de investigação, individual ou grupal, que pretende construir significados a partir dos factos temporais relativos à trajetória pessoal de vida dos sujeitos. Neste tipo de contexto de uso, as histórias de 
vida são frequentemente entendidas como um processo em devir, inacabado, na maior parte do tempo dialógico, que se inscreve no âmbito das relações interpessoais e que implicam sempre distintos tipos de relações de poder, construídas historicamente. Relações de poder traduzidas na trajetória de vida pela filiação, pela ideologia, pela religião, e pela apropriação simbólica de toda uma linguagem social, que ao serem investigadas e explicitadas criticamente dão origem, nos termos da lógica desta abordagem, a um processo educativo e formativo que, trabalhando numa constante passagem entre o registo oral e o registo escrito e entre o passado, o presente e o futuro, adquire as características de uma prática autopoiética, que se inscreve na análise ativa da produção de identidade dos próprios sujeitos, como, alias, a etimologia do termo autopoiesis, do grego autos (eu) e poiein (produzir), sugere (PINEAU; LE GRAND, 1993; LE GRAND, S/D). Um outro traço estruturante comum, nos pressupostos teórico-pedagógicos inerentes a cada uma das terminologias existentes no âmbito da abordagem biográfica e histórias de vida em educação, passa por considerar o especial relevo que é dado à importância que a reflexão crítica deve ocupar no desenvolvimento do processo de educação e formação de jovens e adultos. Trata-se de um aspeto central, muito enfatizado, sobre o qual, por exemplo, Pilon e Desmarais afirmam "a prática das histórias de vida em contexto de formação, como processo de construção identitário e como caminho de emancipação não se atinge de maneira espontânea e natural" (PILON; DESMARAIS, 1996, p. 12). Daí que se considere, de um modo geral, que as práticas assentes em histórias de vida conduzidas reflexivamente "proporcionam uma ocasião de revalorização epistemológica da noção de experiência, e de uma aprendizagem fundada na experiência" (id., ibid., p.13).

Esta lógica geral, que caracteriza a atual utilização das histórias de vida em educação e formação, tem conquistado um espaço cada vez mais significativo no campo da educação e formação de jovens e adultos, sendo possível identificar duas tendências principais, resultantes do mapeamento do seu uso e teorização no sector, nomeadamente: uma primeira tendência, claramente em expansão na atualidade, relacionada com a orientação e formação profissional para explicitar os saber-fazer adquiridos pelo trabalho: são as designadas 'histórias de vida profissionais'; e uma segunda tendência mais relacionada com a formação e desenvolvimento pessoal para retirar benefício de todos os saber-viver implícitos nas esferas privadas da vida quotidiana: são as denominadas 'histórias de vida existenciais' (PINEAU, 2001).

A este respeito Nóvoa refere que "com o título sugestivo de Vidas das Histórias de Vida, publicou Gaston Pineau, em 1980, um livro que marca o início da utilização sistemática do método (auto)biográfico no âmbito da formação de adultos [desde então] a reflexão em torno da utilização das histórias de vida tem-se enriquecido consideravelmente, dando origem a uma série de estimulantes experiências em vários países da Europa e da América do 
Norte" (NÓVOA, s/d, p. 73). De facto, a rede francófona das histórias de vida, em França e no Canadá, em colaboração com as pesquisas da escola de Genebra, inauguraram uma corrente de utilização das histórias de vida em educação e formação de jovens e adultos, que tem sido aprofundada e expandida, e cujos desenvolvimentos têm contribuído para alargar o corpus teórico deste sector em duas vertentes fundamentais: uma mais preocupada com a construção de uma teoria da formação a partir do sujeito aprendente, e outra que se tem concentrado naquele que tem sido um traço distintivo da escola de Genebra, e que é a conceptualização das histórias de vida enquanto metodologia de investigação e de formação. Como afirma Dominicé "a biografia é um instrumento de investigação e, ao mesmo tempo, um instrumento pedagógico (...) esta dupla função da abordagem biográfica caracteriza a sua utilização em ciências da educação" (DOMINICÉ, s/d, p. 78).

Recentemente, assiste-se, portanto, à construção de uma teoria da educação e formação a partir do sujeito aprendente (NÓVOA; FINGER, 1988). Como afirma Nóvoa "as histórias de vida e o método (auto)biográfico integram-se no movimento atual que procura repensar as questões da formação, acentuando a ideia de que 'ninguém forma ninguém' e que 'a formação é inevitavelmente um trabalho de reflexão sobre os percursos de vida" (NÓVOA, s/d, p. 73). Ora, um dos principais contributos para a elaboração desta teoria, com expressão sobretudo nos países de tradição francófona, deriva das formulações elaboradas por Pineau ao longo da sua obra, que explorou teoricamente esta nova dimensão da abordagem biográfica inscrevendo-a nalguns dos pressupostos herdados da perspetiva da educação permanente (BARROS, 2012). No essencial, no pensamento de Gaston Pineau as histórias de vida são conceptualizadas como um método de investigação-ação que procura estimular a autoformação, isto na medida em que o exercício pessoal de explicitação crítica de uma dada trajetória de vida, ou seja, a apropriação retrospetiva do percurso de vida do indivíduo-sujeito, obriga-o a um trabalho de grande implicação, contribuindo para uma tomada de consciência crítica, individual e coletiva, e para uma participação efetiva no seu próprio processo de formação (PINEAU, 1977; 2001).

A utilização das histórias de vida em educação e formação de jovens e adultos, nos termos da conceptualização proposta por Pineau, permite articular no mesmo processo educativo as atividades de formação, de investigação e de intervenção, como defende Dominicé (1990) quando elabora teoricamente acerca do que designou de processo de 'formação-investigação', ou Barbier (1988) no seu processo de 'investigação-formação existencial', reconhecendo-se, em ambos os processos, o aprendente como um produtor de saber, mais do que um consumidor de saber. A este respeito Couceiro considera que a abordagem biográfica e as histórias de vida, ao serem reconhecidas como produtoras de conhecimento, nomeadamente de "um conhecimento sobre si, das relações do sujeito com o processo formativo, um conhecimento 
do modo como o sujeito se tornou e é nas múltiplas dimensões da sua existência" (COUCEIRO, 2000, p. 140), permite recolocar no campo de reflexão da educação e formação as questões: "o que é o conhecimento? Qual a sua natureza? como se constrói o conhecimento?” (id., ibid.).

De igual modo, há a sublinhar que um dos principais e efetivos contributos para a conceptualização e disseminação das histórias de vida enquanto metodologia de investigação e de formação resulta dos trabalhos desenvolvidos no âmbito dos pressupostos da escola de Genebra, e que, segundo Josso, têm girado em torno de dois eixos principais que se influenciam mutuamente: um primeiro eixo relacionado com o "projeto teórico de uma compreensão biográfica da formação, e a fortiori da autoformação, através das perspetivas de investigação-formação" (JOSSO, 2002, p. 15); e um segundo eixo mais debruçado sobre o "uso de abordagens biográficas postas ao serviço de projetos (projeto de expressão, projeto profissional, projeto de reinserção, projeto de formação, projeto de transformação de práticas, projeto de vida)" (id., ibid.).

Sendo assim, e no âmbito do primeiro eixo, que concebe as histórias de vida enquanto projetos de conhecimento, sobressaem especialmente os esforços de teorização realizados para o apuramento de metodologias de investigação-formação que, conforme sublinha Josso, "tinham e continuam a ter necessidade de reivindicar, de criar um espaço, de justificar a sua fundamentação [com o intuito de] conceder legitimidade à mobilização da subjetividade como modo de produção do saber e à intersubjetividade como suporte do trabalho interpretativo e de construção de sentido para os autores das narrativas das histórias de vida" (JOSSO, 2002, p.16), exprimindo, esta preocupação metodológica, sobretudo o desafio epistemológico de refletir acerca do valor de uso dos conhecimentos produzidos e das normas de legitimação de um determinado saber científico; enquanto que, e no âmbito do segundo eixo, que se debruça sobre a abordagem biográfica ou experiencial colocada ao serviço de lógicas de projeto, sobressai por sua vez o carácter mais circunscrito e em muitos casos também mais instrumentalizado dado à narrativa biográfica, uma vez que "as histórias de vida postas ao serviço de um projeto são necessariamente adaptadas à perspetiva definida pelo projeto no qual elas se inserem" (JOSSO, 2002, p. 20). Destacam-se neste eixo dois contextos de uso principais: um mais relacionado com a formação profissional, e outro mais relacionado com o reconhecimento de saberes adquiridos.

Estes dois eixos estruturantes da conceptualização das histórias de vida, enquanto metodologia de investigação e de formação, têm-se traduzido progressivamente numa reflexão, de cariz inovador no panorama do corpus teórico da educação e formação de jovens e adultos, que se tem debruçado, crescentemente, sobre os papéis do autor, ator e leitor na prática de construção e de compreensão das histórias de vida, bem como sobre o poder transformador das mesmas. Neste contexto de teorização aceita-se, de modo con- 
sensual, que a narrativa de vida não tem em si qualquer poder transformador, uma vez que esse poder reside apenas, como sublinha Josso, na "metodologia de trabalho sobre a narrativa de vida [que pode representar a] oportunidade de uma transformação segundo a natureza das tomadas de consciência que aí são feitas e o grau de abertura à experiência das pessoas envolvidas no processo" (JOSSO, 2002, p. 116).

\section{CONSIDERAÇÕES FINAIS NUM DEBATE EM ABERTO}

A prática de investigação-formação assente nas histórias de vida, ou seja, todo o trabalho de reflexão crítica sobre o percurso de vida e sobre a forma como cada um constrói a sua própria narrativa biográfica, deverá possibilitar um trabalho em grande medida propiciador de uma progressiva tomada de consciência dos diversos referenciais teóricos que funcionam como a priori nos processos de conhecimento.

Convocámos, no contexto deste Dossier temático sobre TrabalhoEducação, estas breves diacríticas inerentes à corrente das histórias de vida em educação e formação porque nos parece claro que as mesmas são relevantes para a pós-graduação brasileira (stricto sensu), e não só, na medida em que permitem operar uma significativa deslocação paradigmática no modo de conceber a educação e formação, sobretudo porque se transforma o aprendente num coprodutor da sua educação e formação, e se muda a forma de conceber o trabalho educativo-formativo, que passa, assim, a incidir muito menos na aquisição de conteúdos, para, ao invés, incidir muito mais na compreensão do meio ambiente físico e social. Trata-se de uma abordagem teórico-pedagógica que, partindo dos contributos do movimento das histórias de vida nas ciências sociais, conceptualiza questões centrais para a valorização da experiência de vida e de trabalho na educação e formação convocando para a discussão a contraposição entre o diagnóstico de necessidades e o reconhecimento de adquiridos. Uma problemática que instaurou um debate nascido da tentativa de superação de uma lógica clássica do trabalho educativo-formativo, que, em muitos casos, ainda opera segundo o pressuposto de uma dupla rutura, por um lado, com a experiência dos sujeitos, por outro lado, com os contextos em que se desenrola a sua vida.

\section{REFERÊNCIAS}

BAKER, A.C., JENSEN, P.J.; KOLB, D.A. Conversational learning: an experiential approach to knowledge creation. USA: Greenwood Publishing Group, 2002. 
BARBIER, R. Culture d'Hôpital et Recherche-Formation Existentielle à l'Écoute des Mourants. Pratiques de Formation/Analyses. Culture d'Entreprise et Formation, 101-122, 1988.

BARBIER, J.-M. ; LESNE, M. L'Analyse des Besoins en Formation. Paris: Robert Jauze, 1986.

BARROS, R. From Lifelong Education to Lifelong Learning: Discussion of some effects of today's neoliberal policies. RELA - European Journal for Research on the Education and Learning of Adults, Vol. 3, No.2, 119-134, 2012. http://www.rela.ep.liu.se/article.asp?DOI=10.3384/rela.2000-7426.re$\underline{\mathrm{la} 0071}$

BARROS, R. Genealogia dos Conceitos em Educação de Adultos: Da Educação Permanente à Aprendizagem ao Longo da Vida - Um estudo sobre os fundamentos político-pedagógicos da prática educacional. Lisboa: Chiado Editora, 2011a. Disponível em: http://hdl.handle.net/ $\underline{10400.1 / 14097}$

BARROS, R. A Criação do Reconhecimento de Adquiridos Experienciais (RVCC) em Portugal - Uma Etnografia Crítica em Educação de Adultos. Lisboa: Chiado Editora, 2011b. Disponível em: http://hdl.handle.net/ $\underline{10400.1 / 14093}$

BARROS, R.; LIMA, P. ; AZEVEDO, M. (Org.) (2020). Rumos da Educação e Formação de Jovens e Adultos em Portugal e no Brasil - um balanço comparado de políticas e práticas. Natal: Editoria IFRN-Brasil, 2020. Disponível em : https://memoria.ifrn.edu.br/handle/1044/1835

BAUMAN, Z. Modernidade Líquida. Brasil: Jorge Zahar Editora, 2001.

BEARD, C.; WILSON, J.P. (eds). The power of experiential learning: a handbook for trainers and educators. London: Kogan Page, 2002.

BOLIVAR, A.; DOMINGO, J.; FERNÁNDEZ, M. La investigación biográfica narrativa en educación: enfoque y metodología. Madrid: La Muralla, 2001.

CANÁRIO, R. Educação de Adultos - Um Campo e uma Problemática. Lisboa: Educa-Formação/ANEFA, 2000.

COUCEIRO, M. L. P. Autoformação e Co-formação no Feminino - Abordagem existencial através de Histórias de Vida. Lisboa: Universidade Nova de Lisboa (Tese de Doutoramento não publicada), 2000.

DIONNE, H. Récit Collectif d'une Pratique de Résistance: Recherche-Intervention dans un Village Québécois. In Danielle Desmarais \& Jean-Marc Pilon (coords.). Pratiques des Histoires de Vie - Au Carrefour de la Formation, de la Recherche et de I'Intervention (pp. 179-198). Paris : Édition L'Harmattan, 1996.

DOMINICÉ, P. L'Histoire de Vie Comme Processus de Formation. Paris: Édition L'Harmattan, 1990.

DOMINICÉ, P. A Abordagem Biográfica Enquanto Opção Metodológica. In Educação de Adultos - Cadernos de Formação (pp.75-83). Lisboa: De- 
partamento da Educação Básica/Núcleo de Educação Recorrente e Extra-Escolar, s/d.

GIDDENS, A. The Consequences of Modernity. Oxford: Polity Press, 1991.

ITIN, C.M. Reasserting the philosophy of experiential education as a vehicle for change in the 21st century, Journal of Experiential Education, 22(2), pp. 91-98, 1999.

JOSSO, M-C. Experiências de Vida e Formação. Lisboa: Educa-Formação, 2002.

KOLB, D. Experiential Learning: Experience as the Source of Learning and Development. New Jersey: Prentice-Hall,1984.

LE GRAND, J.-L. Definir les Histoires de Vie - Sus et Insus 'Définotionnels'. Paris: Crise/Laboratoire des Sciences de l'Éducation, s/d.

MALINEN, A. Towards the Essence of Adult Experiential Learning: A Reading of the Theories of Knowles, Kolb, Mezirow, Revans and Schon. Finland: University of Jyvaskyla, 2000.

MIETTINEN, R. The concept of experiential learning and John Dewey's theory of reflective thought and action, International Journal of Lifelong Education, 19(1), pp. 54-72, 2000.

MOON, J. A. Handbook of Reflective and Experiential Learning Theory and Practice. UK: Routledge Falmer, 2004.

NÓVOA, A. Prefácio. In Marie-Christine Josso, Experiências de Vida e Formação (pp. 7-12). Lisboa: Educa-Formação, 2002.

NÓVOA, A. Histórias de Vida. In Educação de Adultos - Cadernos de Formação (pp. 62-74). Lisboa: Departamento da Educação Básica/Núcleo de Educação Recorrente e Extra-Escolar, s/d.

NÓVOA, A.; FINGER, M. O método (auto)biográfico e a formação. Lisboa: Ministério da Saúde, 1988.

PILON, J.-M. ; DESMARAIS, D. Les Enjeux Liés à la Pratique des Histoires de Vie au Carrefour de la Formation des Adultes, de la Recherche et de l'Intervention. In Danielle Desmarais \& Jean-Marc Pilon (Coords.). Pratiques des Histoires de Vie - Au Carrefour de la Formation, de la Recherche et de l'Intervention (pp. 11-24). Paris : Édition L'Harmattan, 1996.

PINEAU, G. Experiências de Aprendizagem e Histórias de Vida. In Philippe Carré \& Pierre Caspar (Dir.). Tratado das Ciências e das Técnicas da Formação (pp. 327-350). Lisboa: Instituto Piaget, 2001.

PINEAU, G. Éducation ou Aliénation Permanente? - repères mythiques et politiques. Paris: Bordas, 1977.

PINEAU, G. ; LE GRAND, J.-L. Les Histoires de Vie. Paris: PUF, 1993.

SILBERMAN, M. (Ed.) The Handbook of Experiential Learning. USA: John Wiley \& Sons, 2007. 
SOUSA SANTOS, B. A Crítica da Razão Indolente - Contra o Desperdício da Experiência. Porto: Edições Afrontamento, 2000.

TOURAINE, A. O Retorno do Actor - Ensaio Sobre Sociologia. Lisboa: Instituto Piaget, 1984.

WESSELS, M. Experiential Learning. Cape Town: Juta Academic, 2006. 